\title{
CAMBIO CLIMÁTICO Y DINÁMICA DE LOS ECOSISTEMAS FORESTALES
}

\author{
CLIMATE CHANGE AND DYNAMICS \\ OF FOREST ECOSYSTEMS
}

\section{Armando Gómez-Guerrero*, Arian Correa-Díaz² y Luis U. Castruita-Esparza ${ }^{3}$}

\author{
'Colegio de Postgraduados, Posgrado en Ciencias Forestales, Montecillo, Texcoco, Estado de México, México. ${ }^{2}$ Instituto Nacional de Investigaciones \\ Forestales, Agrícolas y Pecuarias, Centro Nacional de Investigación Disciplinaria en Conservación y Mejoramiento de Ecosistemas, Coyoacán, \\ Ciudad de México, México. ${ }^{3}$ Universidad Autónoma de Chihuahua, Delicias, Chihuahua, México. \\ *Autor de correspondencia (agomezg@colpos.mx)
}

\section{RESUMEN}

Para comprender el comportamiento de los bosques ante el cambio climático es importante tomar en cuentan los procesos asociados con la funcionalidad de los ecosistemas forestales. En este trabajo de revisión se describen de manera general las tendencias en los cambios de la superficie forestal en México, destacando la capacidad de recuperación natural de los ecosistemas forestales. Dada la estrecha relación de los bosques y el agua, se incluye la relación de cambio de cobertura y escurrimiento superficial. También se destacan las principales perturbaciones de los bosques como consecuencia del cambio climático. Como aportación más relevante se enfatizan cinco procesos que son poco considerados en el tema de cambio climático, pero que definen la respuesta dinámica de los ecosistemas arbolados: la deficiencia hidráulica, eficiencia de uso de agua, la xilogenésis, los componentes de resiliencia e histéresis del ecosistema. La comprensión de estos puntos críticos ayuda en el entendimiento de la respuesta de los ecosistemas forestales ante el cambio climático. Una explicación científica del funcionamiento de los ecosistemas forestales ayudará a proponer estrategias específicas sobre manejo de estos ecosistemas y generar modelos con mayor capacidad de predicción de la dinámica de los bosques ante el cambio climático.

Palabras clave: Cambio climático, ciclo del carbono, deforestación, funcionalidad de ecosistemas forestales, productividad en bosques.

\section{SUMMARY}

To understand how forests will behave under climate change, it is important to consider the processes related to the functionality of forest ecosystems. In this review the trends of the forest area of Mexico is described in a general context, highlighting the natural recovery capacity of forest ecosystems. Given the strong connection between forests and water, the relationship between changes in cover and surface runoff is addressed. The main disturbances to forests as a consequence of climate change are also highlighted. As the most relevant contribution, five critical process are emphasized that are seldom considered in the subject of climate change, but these process define the dynamics of forests ecosystems, namely: hydraulic failure, water use efficiency, xylogenesis, resilience components and ecosystem hysteresis. Understanding these critical points helps in understanding the response of forest ecosystems to climate change. A scientific explanation of how forest ecosystems work will help to propose specific strategies on the management of these ecosystems and generate models with greater and more realistic capacity to predict forest dynamics under the scenario of climate change.
Index words: carbon cycle, climate change, deforestation, forest productivity, functionality of forest ecosystems.

\section{INTRODUCCIÓN}

Los bosques juegan un papel clave en el flujo de bióxido de carbono de la vegetación y suelo hacia la atmósfera (Schlesinger y Bernhardt, 2013), representando un almacén de carbono (biomasa y suelo) más grande que el de la atmósfera, a pesar de su limitada superficie ( $\approx 30 \%$ de la superficie terrestre) (Pan et al., 2011); además, los bosques influyen en el flujo energético global mediante su albedo, que es afectado por la deforestación y cambios en la composición de especies. El factor de albedo de los bosques en verano oscila entre 0.20 y 0.28 , influyendo en la absorción de energía global (Otto et al., 2014). La relación entre la temperatura y la emisión de energía por radiación se puede explicar por la ley de Stefan-Boltzman, la cual ayuda a comprender la importancia del tipo de uso de suelo (natural vs. antropogénico) en la variación de la temperatura a nivel global (Schlesinger y Bernhardt, 2013).

El $\mathrm{CO}_{2}$ atmosférico ha incrementado en las últimas décadas, llegando actualmente a 410 ppm, con importantes implicaciones para el funcionamiento de los ecosistemas (Yang et al., 2019). Anteriormente se había considerado que el incremento en la concentración de $\mathrm{CO}_{2}$ atmosférico podría traducirse en una mayor productividad de los bosques, debido a que el $\mathrm{CO}_{2}$ es un insumo esencial de la fotosíntesis; de hecho, algunos estudios han reportado un incremento en el índice de área foliar ("enverdecimiento") de diferentes ecosistemas a nivel global, atribuible a un efecto de fertilización de $\mathrm{CO}_{2}$ a partir de la década de los años 80s del siglo anterior (Piao et al., 2020; Zhu, et al., 2016); sin embargo, información reciente indica que tanto el beneficio de mayor concentración de $\mathrm{CO}_{2}$ atmosférico como mayor de depósito de $\mathrm{N}$ se verá contrabalanceado con el aumento de temperatura y las sequias (Duffy et al., 
2021; Peñuelas et al., 2017). No obstante, la asimilación de $\mathrm{CO}_{2}$ por las plantas es un proceso complejo que depende de varios factores que pueden ser de tipo enzimático o físico, pero también regido por leyes de intercambio de gases entre la hoja y la atmósfera, así como otros factores relacionados al estado hídrico y nutricional de los árboles (Voelker et al., 2016).

Existen diferentes enfoques para evaluar los efectos del incremento de $\mathrm{CO}_{2}$ en los ecosistemas forestales, entre ellos, los experimentos en cámaras de crecimiento (con altas concentraciones de $\mathrm{CO}_{2}$ ) indican un cierre estomático de las plantas al aumentar la concentración de $\mathrm{CO}_{2}$. El comportamiento estomático en los árboles tiene profundas implicaciones sobre los procesos de intercambio de gases y la eficiencia de uso de agua en la vegetación (Battipaglia et al., 2013). A pesar de que los efectos del cambio climático en bosques se han centrado en la acumulación de biomasa y el ciclo del carbono, el resultado final depende de varios factores funcionales a nivel de comunidad arbórea. Si no se comprenden dichos factores no se pueden plantear estrategias específicas para la conservación de las zonas forestales.

El objetivo de la presente revisión es dejar una reflexión sobre la importancia del funcionamiento de los ecosistemas forestales y las consecuencias ante la variabilidad climática y de la composición atmosférica. Es crítico considerar otros procesos que normalmente no se incluyen en el estudio de la respuesta de los bosques ante el cambio climático, tales como la deficiencia hidráulica, eficiencia de uso de agua, xilogenésis (formación de la madera), componentes de resiliencia e histéresis del ecosistema. La comprensión de los puntos anteriores estimulará a los investigadores a considerar otras variables en sus modelos de predicción para mejorar el entendimiento de la respuesta de los bosques ante el cambio climático.

\section{DINÁMICA DE LA COBERTURA ARBOLADA}

De acuerdo con estimaciones de Hansen et al. (2013), en el periodo de 2000 a 2012 se perdieron 230 millones de ha arboladas en el mundo, aunque también se restablecieron 80 millones de ha por un efecto de recuperación natural de la vegetación y el establecimiento de áreas arboladas. Lo anterior corresponde a una pérdida anual de 19.2 millones de ha y una recuperación de 6.67 millones de ha, con un cambio neto anual de -12.33 millones de ha. El ecosistema con mayor pérdida en superficie arbolada fue el bosque tropical; sin embargo, la pérdida de superficie arbolada es difícil de estimar porque la vegetación forestal es dinámica, ya que al mismo tiempo que se pierde superficie en un lugar, en otras áreas hay recuperación, ya sea en forma natural o por la influencia del hombre (Curtis et al., 2018).
En Latinoamérica, la recuperación natural de bosques tropicales secundarios jóvenes (menores de 20 años) y maduros (20-60 años) podría mitigar considerablemente las pérdidas de carbono, debido a que su tasa de acumulación de biomasa es entre 1.2 y 2 veces mayor que en los bosques viejos (Chazdon et al., 2016).

Los datos de Hansen et al. (2013) constituyen un esfuerzo global en el que es posible medir la capacidad de recuperación de la superficie arbolada. Con estos datos se puede analizar la capacidad de recuperación de superficie arbolada en diferentes países (Figura 1); sin embargo, es importante aclarar que la dinámica está influenciada por distintos factores en cada país; por ejemplo, en Suecia y Estados Unidos de América la recuperación de superficie arbolada se debe a la aplicación de métodos silvícolas. En Malasia la pérdida de superficie arbolada se debe principalmente a cambio de uso de suelo (Masum et al., 2017; Varkkey et al., 2018), y su recuperación es el reflejo de procesos naturales. Aunque de acuerdo con los metadatos analizados por Hansen et al. (2013), México está dentro de los 15 países con mayor pérdida neta de superficies arbórea, los datos muestran una capacidad moderada de recuperación, equivalente al $26 \%$, que es el resultado de la recuperación natural y plantaciones forestales.

La pérdida anual estimada entre 2000 y 2012 para México fue de 198,850 ha, con una recuperación de 52,775 ha (Hansen et al., 2013), lo que representa una tasa media anual de $-0.21 \%$, mayor a la tasa a nivel global (-0.12) entre 1990 y 2015 (Keenan et al., 2015), aunque se estima que recientemente esta tasa en México ha disminuido a valores de entre -0.13 y $-0.14 \%$, lo que constituye un avance significativo en la estrategia de conservación forestal (CONAFOR, 2019; FAO, 2020). La dinámica en la superficie forestal México refleja la tendencia a nivel global, afectando principalmente a ecosistemas tropicales. A nivel global, la pérdida de cobertura forestal es consecuencia del cambio de uso de suelo con fines de agricultura, minería e infraestructura para la producción de energía, además de deforestación, agricultura itinerante e incendios forestales (Curtis et al., 2018). La superficie arbolada de México en el escenario global es muy importante, ya que sobre la franja latitudinal donde se ubica este país, sólo existe otra región arbolada importante, que corresponde a Tailandia.

\section{LOS BOSQUES Y EL AGUA}

La relación entre los bosques y la producción de agua es amplia y difícil de separar. Los bosques contribuyen al ciclo hidrológico a través de la evapotranspiración e infiltración; además, proveen un efecto regulador de temperatura (Bonan, 2008). Si el cambio climático reduce y degrada las áreas forestales, la provisión de servicios hidrológicos 
también está en riesgo (Gómez-Guerrero y Doane, 2018); por ejemplo, el horizonte orgánico superficial del suelo forestal no sólo disminuye la erosión, sino también actúa como un filtro que incrementa la calidad del agua. La importancia del piso orgánico forestal en México se minimiza a tal grado que se comercializa la "tierra de monte" como un producto del bosque avalado por la regulación forestal (Mantero et al., 2019).

Dentro de una cuenca hidrográfica, además de la población humana, el sector agrícola, industrial y de servicios (principalmente turismo) dependen de los recursos hídricos generados por el bosque (Zhang, et al., 2017). En general, con la pérdida de superficie arbolada, el escurrimiento superficial aumenta, especialmente en cuencas grandes, superiores a $1000 \mathrm{~km}^{2}$; en cambio, el efecto de la ganancia de cobertura sobre la producción de agua es más complejo, ya que no siempre muestra efectos favorables, debido a la tasa de transpiración de las especies (Zhang et al., 2017).

La variación en la precipitación influye tanto en el crecimiento del arbolado como en el caudal medio de una cuenca (Villanueva et al., 2017). Esta relación estrecha permite la correlación entre crecimiento de los anillos anuales en especies forestales y el aforo de una cuenca. La ventaja de establecer una correlación entre la dinámica de anillos y la producción de agua estriba en que se puede establecer una línea base en las cuencas y valorar los efectos indirectos del cambio climático por la pérdida de vegetación (Villanueva-Díaz et al., 2005). Los años con mayor precipitación y mayores volúmenes de caudal medio en los ríos correlacionan con los índices de crecimiento de los anillos (Chen et al., 2020).

Por otra parte, dado que la información histórica del caudal medio en una cuenca es limitada, las reconstrucciones a partir de los anillos de crecimiento permiten estimar la variabilidad en plazos largos y deducir si los efectos de cambio climático se han manifestado en la cuenca. La resiliencia del escurrimiento superficial en una cuenca forestal es mayor cuando los bosques son mixtos (Zhang et al., 2017); por lo tanto, las estrategias silvícolas que preserven la diversidad de especies forestales son más conveniente para amortiguar la escasez de agua de años secos y los disturbios del cambio climático.

\section{LOS DISTURBIOS DEL CAMBIO CLIMÁTICO}

De acuerdo con predicciones recientes del IPCC, el calentamiento global con respecto al periodo preindustrial (1850-1900) corresponderá a un incremento en temperatura de $1.5^{\circ} \mathrm{C}$, asumiendo que la emisiones netas de $\mathrm{CO}_{2}$ se igualan a cero en el año 2055 y el forzamiento radiativo de otros gases de efecto invernadero se reduce a partir de 2030 (IPCC, 2018); sin embargo, es importante decir que para que se cumpla el escenario mencionado se requiere, entre otras cosas, que las áreas forestales se cultiven con métodos sustentables y la forestación y reforestación se realicen a grandes escalas. Por lo anterior,

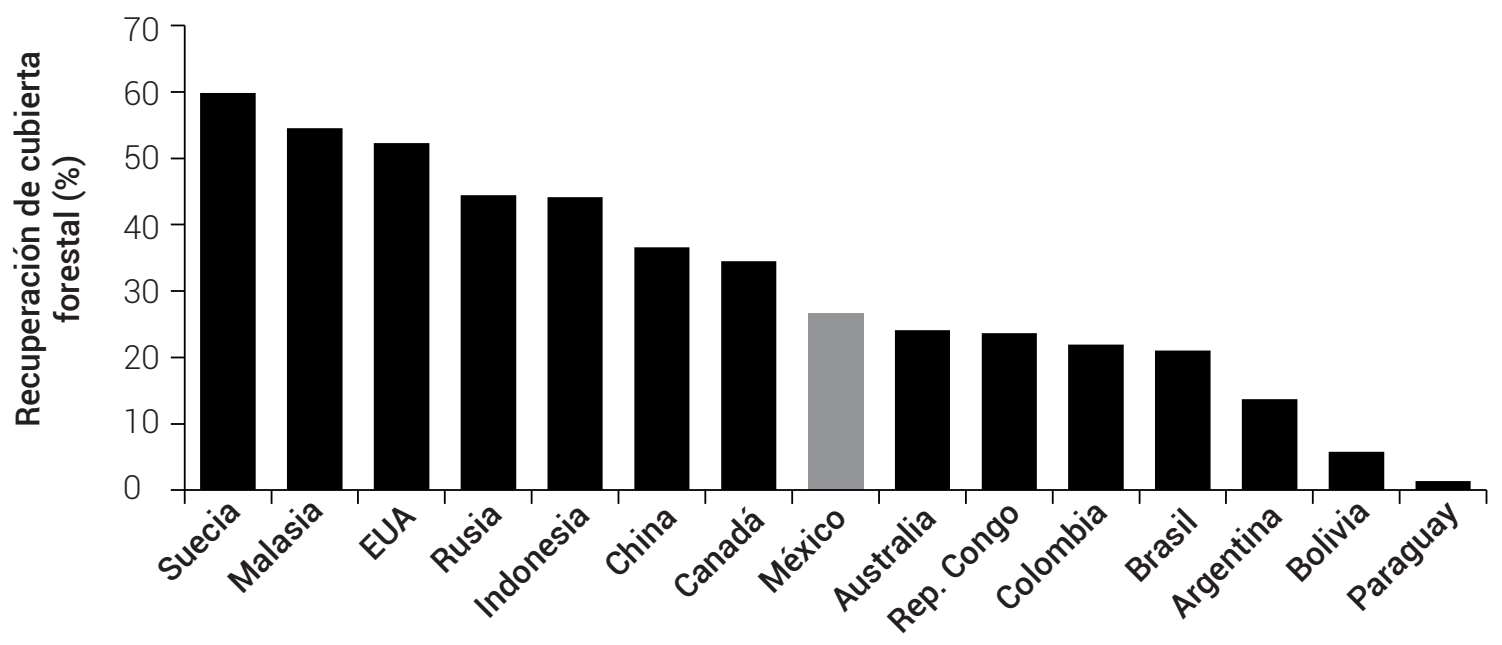

Figura 1. Tasa de recuperación de cubierta forestal estimada para diferentes países. Los cálculos se realizaron a partir de la información complementaria de Hansen et al. (2013). La recuperación en países desarrollados es un reflejo principalmente de los avances de la silvicultura, mientras que en otros se explica por la recuperación natural combinada con actividades de reforestación y forestación. 
la dinámica de las superficies arboladas y su capacidad de amortiguamiento son importantes ante el cambio climático. Aun cuando no se ha alcanzado el incremento global de $1.5^{\circ} \mathrm{C}$, los efectos negativos del cambio climático sobre los ecosistemas terrestres y la pérdida de los servicios ambientales que proveen ya se han empezado a percibir (IPCC, 2018).

Seidl et al. (2017) realizaron una revisión exhaustiva de los principales disturbios en los ecosistemas forestales como consecuencia del cambio climático, destacando como principales agentes de disturbio a las enfermedades, fuego, sequía, viento, bajas temperatura e insectos. Las regiones secas y con mayor temperatura son vulnerables a la sequía, y como consecuencia, al ataque de insectos, mientras que en las regiones húmedas la vulnerabilidad a las enfermedades y daños al arbolado por vientos fuertes aumenta. La mayor superficie afectada ha sido el bosque boreal, donde los eventos extremos de sequía terminan con la muerte masiva de arbolado por el ataque de insectos (Seidl et al., 2017). Generalmente se asume que en la medida que se acentúe el aumento de temperatura por efecto del cambio climático las especies forestales migrarán de las zonas/latitudes bajas a las zonas/latitudes altas; sin embargo, la humedad parece ser una variable más determinante que la temperatura, y la migración del este al oeste en bosques de los Estados Unidos ha corroborado esta tendencia (Fei et al., 2017). Parece improbable que las poblaciones de especies forestales logren migrar por medios naturales a la velocidad que está modificándose espacialmente el hábitat climático que le es propicio. La predicción del desplazamiento latitudinal y altitudinal de las especies forestales es muy compleja y difícilmente hay proporciones de cambio por unidad de cambio altitudinal que correspondan con un determinado movimiento latitudinal. En sentido altitudinal los cambios en temperatura, presión parcial de los gases y la calidad de la radiación solar son más abruptos (Jump et al., 2009).

\section{SEQUÍA Y DEFICIENCIA HIDRÁULICA}

La muerte masiva de arbolado, como consecuencia del cambio climático se detona debido a sequías extremas y altas temperaturas (Choat et al., 2018). La mortalidad extendida de arbolado ya está ocurriendo en todas partes del mundo y es muy probable que la vulnerabilidad de las especies forestales, y por lo tanto la superficie afectada, se estén subestimando debido a la falta de información y a la no linealidad que se presentan en algunas variables como la humedad atmosférica (Allen et. al., 2015). Algunos autores han sugerido que la muerte de arbolado por sequías puede explicarse con la ecuación de conductividad hidráulica propuesta por Darcy (McDowell y Allen, 2015). Esta analogía permite explicar la muerte del arbolado, ya que el movimiento del agua dentro del xilema lo determina un gradiente de potencial hídrico y propiedades intrínsecas que corresponden al sistema de conducción de cada especie. De esta forma, el principio puede explicarse con la siguiente ecuación propuesta por McDowell et al. (2008) y McDowell y Allen (2015).

$$
G=\frac{\operatorname{Asks}\left(\psi_{S}-\psi_{l}\right)}{h \eta A / D}
$$

donde G: conductancia estomática, As: área de conducción (albura), ks: conductividad (xilema), $\psi_{\text {s: }}$ potencial hídrico suelo, $\Psi_{1}$ : potencial hídrico hoja, h: altura del árbol, n: viscosidad del agua, Al: area foliar, D: déficit de presión de vapor.

De acuerdo con la ecuación (1), es evidente que si ocurre un incremento en la temperatura, como se tiene pronosticado, D aumentará, por lo que para mantener la conductancia estomática y al mismo tiempo soportar el aumento de $\mathrm{D}$, los árboles que minimicen la altura y área foliar serán más aptos para enfrentar el cambio climático; por ejemplo, Stovall et al. (2019) reportaron que durante eventos de sequía extrema los árboles altos (> $30 \mathrm{~m}$ ) mueren a una tasa dos veces mayor que los árboles pequeños $(<15 \mathrm{~m})$.

De la misma forma, los árboles que puedan soportar mayor diferencial de potencial entre el suelo y la hoja (anisohídricos) serán más aptos para enfrentar el cambio climático al mantener la tasa de conductancia estomática ante aumentos del déficit de presión de vapor. La ecuación de Darcy permite anticiparse a diferentes escenarios; sin embargo, es importante comprender al árbol como un sistema y las variables que definen su estado hídrico. La explicación de McDowell y Allen (2015) es útil para comprender la deficiencia hidráulica, que es un mal funcionamiento del xilema para conducir el agua, y que ocurre en los árboles bajo sequías extremas, y explica también por qué los árboles manifiestan inicialmente evidencias de estrés hídrico, con la punta seca y la pérdida de follaje. Cuando la intensidad de sequía es alta se rompe la continuidad hidráulica en el sistema de conducción del árbol, superándose la capacidad de recuperación (Anderegg et al., 2016). Desde esta perspectiva, considerar que en todos los casos los árboles podrían crecer más por los aumentos de $\mathrm{CO}_{2}$ atmosférico es una propuesta incompleta que no considera el funcionamiento estomático y otras variables que determinan el estatus hídrico de los árboles. 


\section{EFICIENCIA DE USO DE AGUA}

El incremento de $\mathrm{CO}_{2}$ atmosférico (Ca) tiene una influencia directa en la fisiología de las plantas (Saurer et al., 2004). El proceso de intercambio de gases entre las hojas y la atmósfera se rige por la ley de difusión de Fick, que señala que la difusión es proporcional a un gradiente de concentración y a una constante de proporcionalidad. Para el caso de las plantas, la asimilación de $\mathrm{CO}_{2}$ se expresa de la siguiente forma:

$$
A=g_{s}\left(C_{a}-C_{i}\right)
$$

donde $A$ representa la asimilación de bióxido de carbono, $g_{s}$ es la conductancia estomática y $C_{a}$ y $C_{i}$ son la concentración de $\mathrm{CO}_{2}$ en la atmósfera y al interior de la hoja, respectivamente. En la ecuación (2), el diferencial $C_{a}$ - $C_{i}$ depende de la respuesta de cada especie. De acuerdo con la respuesta de la vegetación, se tienen tres escenarios posibles de respuesta al incremento de $C_{a^{\prime}}$; el primero es que $C_{i}$ se mantenga constante; es decir, que el incremento de $C_{a}$ no influya en la concentración interna de la hoja. El segundo escenario es que el diferencial $C_{a}-C_{i}$ se mantenga constante por una respuesta activa de la vegetación y el tercero que el cociente $C_{i} / C_{a}$ se mantenga constante, también como una respuesta activa de la vegetación. El intercambio de gases entre la hoja y la atmósfera es complejo y requiere de más investigación para entender los efectos del incremento de $C_{a}$ en la vegetación (Xu et al., 2014). Hasta ahora, las respuestas más comunes son de tipo activo, involucrando los escenarios dos y tres, o una combinación de éstos (Voelker et al., 2016). Los experimentos controlados indican que al aumentar $C_{a}$ las plantas tienden a cerrar los estomas, reduciendo su conductancia estomática $\left(g_{s}\right)$. Este efecto se traduce en un ahorro de agua, que a su vez conduce a un aumento en la eficiencia de uso de agua $\left(\mathrm{A} / \mathrm{g}_{\mathrm{s}}\right)$, aunque ello no necesariamente signifique mayor crecimiento (CorreaDíaz et al., 2020a; Peñuelas et al., 2011; Voelker et al., 2016).

A partir de los anillos de crecimiento de especies forestales es posible reconstruir la eficiencia de uso de agua, ya que los fotosintatos de un año en particular producen una firma característica de ${ }^{13} \mathrm{C}$ (isótopo estable de carbono 13). La composición de isótopos estables en anillos de crecimiento es una "huella" natural que sirve como un registro de información del estado hídrico y de la eficiencia de uso de agua en especies forestales en décadas o siglos pasados (Gómez-Guerrero et al., 2013).
Dado que el carbono y oxígeno son dos elementos integrados a la madera durante el crecimiento de los árboles, el análisis de la composición de sus isótopos estables, paralelo con el estudio de los anillos de crecimiento, permite dilucidar sobre los efectos de $C_{a}$ sobre el crecimiento y de estado hídrico del pasado en especies forestales, mientras que el ${ }^{13} \mathrm{C}$ ayuda a identificar eventos de estrés hídrico (sequía) en los anillos de crecimiento, y cambios netos de discriminación de ${ }^{13} \mathrm{C}$ de la atmósfera hacia la hoja, el ${ }^{18} 0$ está relacionado con el proceso de evapotranspiración y apertura estomática (Dawson et al., 2002; Voltas et al., 2013). Por la independencia de ${ }^{13} \mathrm{C}$ y ${ }^{18} \mathrm{O}$ con relación a la fotosíntesis y transpiración, se puede reconstruir el desempeño fisiológico de los árboles a partir de los anillos de crecimiento y deducir el balance entre la ganancia de carbono y la pérdida de agua (Roden y Farquhar, 2012). La eficiencia de uso de agua intrínseca (EUAi), estimada a partir de la composición de isótopos de carbono en la madera, es un indicador fisiológico importante que permite entender la forma en que han respondido las especies forestales a los cambios de $\mathrm{CO}_{2}$ atmosférico en las últimas décadas (Peñuelas et al. 2011). Mediante la estimación de EUAi a partir de ${ }^{13} \mathrm{C}$ se ha encontrado que en bosques alpinos de Pinus hartwegii y Abies religiosa de México el beneficio de mayor EUAi por los incrementos de $\mathrm{CO}_{2}$ atmosféricos no compensa la ganancia en crecimiento, y posiblemente la adquisición de nutrientes y agua del suelo será un factor limitante para capitalizar los beneficios de un efecto de fertilización por $\mathrm{CO}_{2}$ atmosférico (Correa-Díaz et al., 2020a).

La estimación de la eficiencia de uso de agua intrínseca a partir de la composición química en los anillos de crecimiento tiene su base en los fundamentos expuestos por Farquhar et al. (1982), quienes establecieron que la discriminación de ${ }^{13} \mathrm{C}$ en las plantas $\mathrm{C}_{3}$ se expresa por la siguiente ecuación:

$$
\left.{ }^{13} \mathrm{C}=\left(\delta^{13} \mathrm{C}_{\text {aire }}-\delta^{{ }^{13}} \mathrm{C}_{\text {planta }}\right) /\left(1+\delta^{13} \mathrm{C}_{\text {planta }}\right) / 1000\right)
$$

donde: $\triangle^{13} \mathrm{C}$ es la discriminación de ${ }^{13} \mathrm{C} ; \delta^{13} \mathrm{C}_{\text {aire }}$ y $\delta^{13} \mathrm{C}_{\text {planta' }}$ denotan la composición de ${ }^{13} \mathrm{C}$ para la atmósfera (la fuente) y la madera de los anillos (el producto), respectivamente (McCarroll y Loader, 2004). La discriminación de ${ }^{13} \mathrm{C}\left(\triangle^{13} \mathrm{C}\right)$ se expresa en términos de las variables de difusión de la siguiente forma:

$$
\Delta^{13} \mathrm{C}=\mathrm{a}+(\mathrm{b}-\mathrm{a}) \times\left(\frac{\mathrm{C}_{\mathrm{i}}}{\mathrm{C}_{\mathrm{a}}}\right)
$$


donde a representa la discriminación de ${ }^{13} \mathrm{CO}_{2}$ debido a la difusión a través de los estomas $(4.4 \%)$, b es la discriminación neta debida al proceso de carboxilación (27 $\%$ ), y Ca y $\mathrm{Ci}$ las concentraciones de $\mathrm{CO}_{2}$ en la atmósfera y en el interior de la hoja, respectivamente. Finalmente, la EUAi puede ser calculada considerando la diferencia de difusión entre $\mathrm{CO}_{2}$ y vapor de agua $\left(0.625 \mathrm{gs}=\mathrm{gCO}_{2}\right)$, de acuerdo con la ley de Fick.

$$
\text { EUAi }=\frac{A}{g_{2} \mathrm{O}}=C_{a} \times\left[1-\left(\frac{C_{i}}{C_{a}}\right)\right] \times 0.625
$$

La Figura 2 muestra la relación entre la tasa anual EUAi y la temperatura media anual para diferentes especies forestales. Las especies que crecen en ambientes de mayor temperatura tienen una mayor tasa de incremento en EUAi. Esta información sirve como línea base para futuras mediciones que permitan conocer el comportamiento de las especies forestales ente el cambio climático.

\section{XILOGÉNESIS}

La xilogénesis incluye el estudio de los componentes estructurales de la madera y su fenología (Mendivelso et al., 2016). La formación de la madera refleja las ganancias de carbono en el ecosistema. En las coníferas, la formación de anillos de crecimiento es de manera anual, con dos bandas diferenciables entre sí (madera temprana y tardía). En la estación de crecimiento se forman traqueidas de lumen amplio y pared celular delgada (madera temprana), mientras que conforme disminuyen las condiciones ambientales óptimas, las traqueidas se vuelven de menor diámetro y su pared celular se vuelve más gruesa (madera tardía). No existe una separación abrupta entre la madera temprana y la madera tardía, sino una zona de transición (Plomion, et al., 2001). El cambio climático puede afectar el ritmo de formación de la madera y la proporción madera temprana y madera tardía.

La variación en las proporciones de madera temprana y tardía afectan la densidad de la madera, y por lo tanto, la masa neta de carbono acumulada. Existen dos factores relacionados con el cambio climático que pueden afectar la formación de madera o xilogénesis; el primero es la temperatura, que junto con otros procesos bioquímicos activa el cambium, por lo que un aumento de temperatura puede estimular la actividad cambial en las especies forestales; el segundo factor es la duración de la estación de crecimiento, si ésta se prolonga la duración del proceso de formación de madera también se ampliará. Es importante

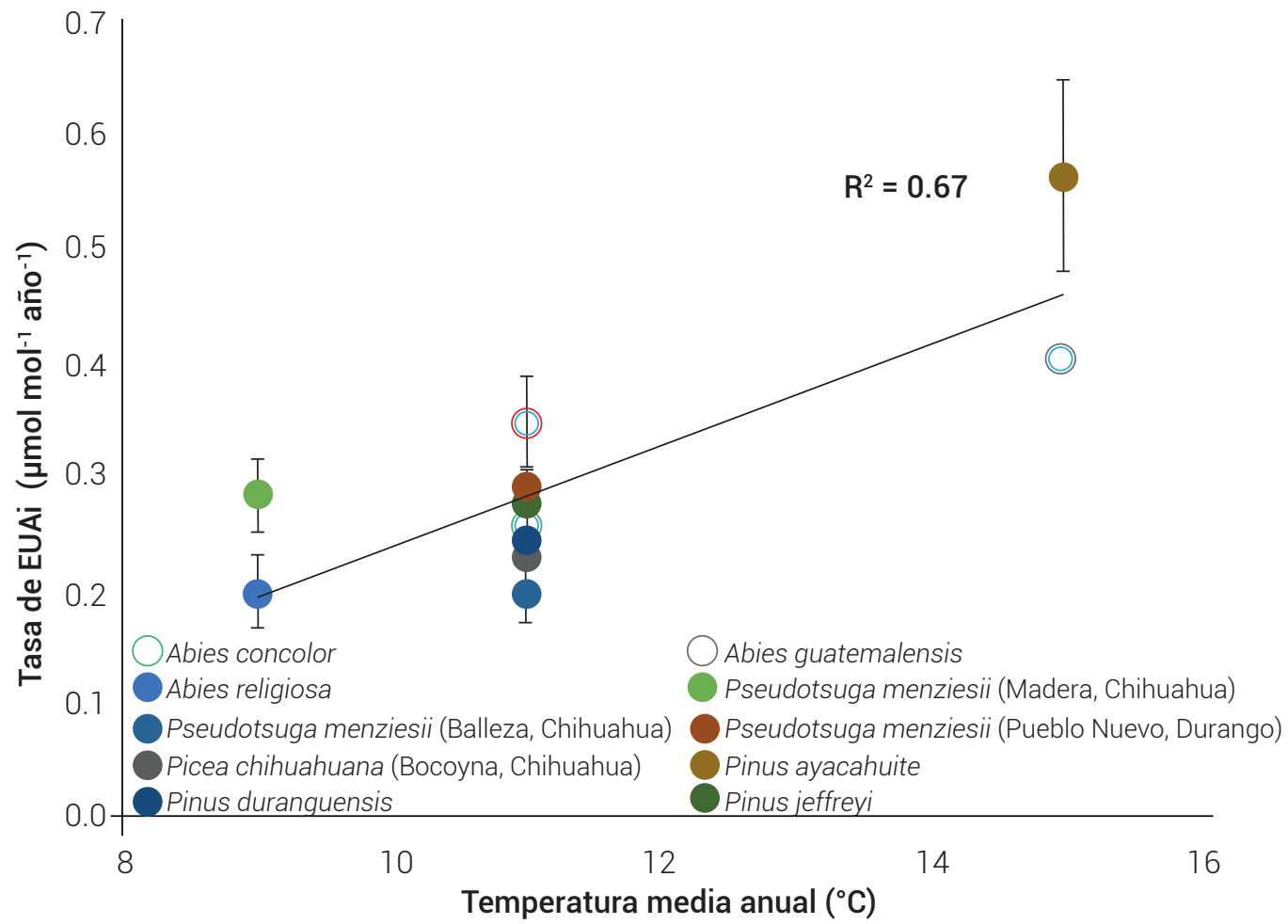

Figura 2. Relación entre la tasa anual de EUAi y la temperatura media anual para diferentes especies forestales. Las especies forestales de climas secos muestran tasas de cambio mayores, información que sirve como referencia para observaciones futuras. 
realizar estudios para saber el grado en que un periodo prolongado de formación de madera estimulará la captura de carbono, ya que en condiciones de mayor temperatura los árboles podrían tener un proceso de respiración más acentuado y dejar de acumular carbono en los componentes subterráneos. La variación de la densidad de la madera implica cambios y ganancia de carbono en la vegetación; por lo tanto, si el cambio climático induce cambios en esta propiedad también ocurrirán cambios en el ciclo del carbono en los ecosistemas forestales (CorreaDíaz et al., 2020b).

Los avances en sensores remotos han facilitado el análisis espacio-temporal en los ecosistemas forestales; por ejemplo, para los bosques de coníferas del centro de México, el vigor del arbolado evaluado con índices de vegetación muestra una clara relación entre la productividad, la actividad estacional de crecimiento y la variación climática (Correa-Díaz et al., 2019); así mismo, también se han estudiado los cambios en la densidad de la madera de acuerdo con la variación topográfica y de microclima en bosques alpinos del centro de México (Corea-Díaz et al., 2020b). El arreglo de los componentes de madera en las especies forestales es un proceso biofísico en el que debe haber eficiencia de soporte y transporte de agua (Begum et al., 2018); por lo anterior, la xilogénesis y el cambio climático tienen una relación estrecha.

\section{COMPONENTES DE RESILIENCIA}

De acuerdo con Lloret et al (2011), los principales índices para examinar la estabilidad de los árboles son la resistencia, recuperación y resiliencia. La resistencia es la habilidad del árbol para oponerse a la reducción en crecimiento y se mide como una relación entre el crecimiento arbóreo durante un disturbio (e.g. sequía) y el crecimiento previo al disturbio. La recuperación es la habilidad de superar el daño después de un disturbio y se mide con el cociente entre el crecimiento posterior al disturbio y el crecimiento durante el disturbio. La resiliencia es la habilidad de alcanzar el nivel de crecimiento que ocurría antes del disturbio y se estima con el crecimiento posterior y antes del disturbio.

Los índices descritos asociados a la resiliencia se pueden estimar en una serie de crecimiento, y para este caso, una serie dendrocronológica puede servir como una referencia (Lloret et al., 2011). La Figura 3, construida con datos de Castruita-Esparza et al. (2016) muestra el crecimiento radial expresado en valores estandarizados de índice de anillo en árboles de Pseudotsuga mensiezii en Chihuahua, México (Gráfica superior, Figura 3). Destacan los años 1893, 1953 y 1996 en los que eventos extremos de sequía llevaron a los árboles a reducir drásticamente su crecimiento. Si se toma como referencia el crecimiento medio histórico
(1.0), se puede decir que, en eventos secos extremos, los árboles resistieron reducciones de crecimiento radial de hasta 66 \% con relación a su crecimiento histórico ( 0.34 en 1953). Su capacidad de recuperación es de hasta 2.9 en términos del índice de ancho de anillo. De la misma forma, su resiliencia sería igual a la unidad, ya que a pesar de los efectos negativos de los eventos extremos, el arbolado pudo recuperar sus tasas de crecimiento histórico; sin embargo, la recuperación después de un evento extremo no siempre se logra en el mismo tiempo. Ante los efectos del cambio climático, los ecosistemas forestales pueden mostrar cierto retardo en la recuperación y en algunos casos ya no es posible recuperar la tasa de crecimiento histórica. A este retardo se le conoce como histéresis del ecosistema. En términos físicos, la histéresis se refiere al desfasamiento en respuesta de un sistema después de haber estado sujeto a un factor de fuerza o magnetismo externo. Para fines de los ecosistemas forestales es el retardo en la recuperación después de un disturbio; por ejemplo, después de un año sequía extrema, aun cuando el siguiente año se estabilice la precipitación, tomará tiempo recuperar el nivel previo de productividad del ecosistema.

\section{HISTÉRESIS DEL ECOSISTEMA}

La histéresis en los ecosistemas forestales ha sido estudiada por Anderegg et al. (2015), observando que la recuperación en crecimiento de los ecosistemas forestales después de un disturbio varía de acuerdo con el clima local y las especies forestales. Los autores analizaron 1,338 cronologías de anillos de crecimiento, de las cuales 695 contaban con datos climáticos asociados. Los resultados indicaron que, en general, existe un retardo medio de cuatro años en la recuperación de los ecosistemas forestales, lo cual es extremadamente importante, ya que en los modelos de predicciones de cambio climático no se considera el componente de histéresis. El legado de los eventos extremos de sequía es mayor en climas áridos y, a nivel de familia, entre Fagaceas y Pinaceas, estas últimas son más susceptibles y menos resilientes (Anderegg et al., 2015). Este hallazgo es relevante para las condiciones de nuestro país, que cuenta con grandes extensiones de bosques de pino y asociaciones de pino-encino, los cuales están creciendo bajo condiciones de baja humedad. Los retardos en la recuperación de ecosistemas forestales son muy importantes para comprender el ciclo del carbono y los efectos del cambio climático sobre el mismo (Anderegg et al., 2015; Kannenberg et al., 2019). Algunos autores han determinado con éxito el umbral (tanto en magnitud como en tiempo) que resisten los árboles bajo condiciones de sequía; por ejemplo, Huang et al. (2015) señalaron que 11 meses consecutivos de sequía extrema son suficientes para desencadenar mortalidad en los árboles; además, se sabe que muerte masiva de arbolado por sequías intensas 

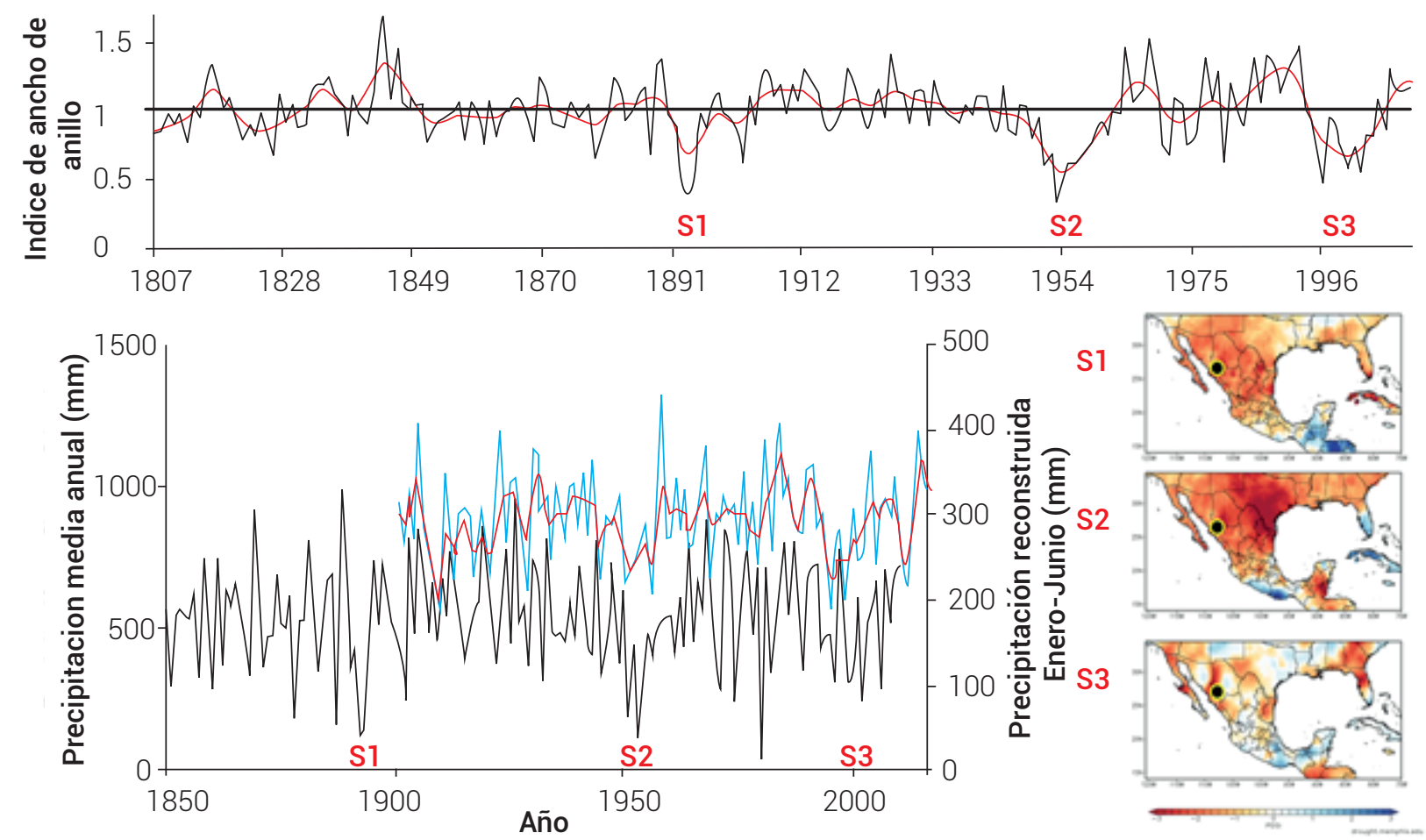

Figura 3. Crecimiento expresado como índice de anillo (gráfica superior) de Pseudotsuga mensiezii en la Sierra Madre Occidental. Los extremos de bajo crecimiento (S1, S2 y S3) corresponden con los períodos de baja precipitación (gráfica inferior) y con los índices de sequía de Palmer (PDSI) de acuerdo con el Atlas de Sequia Mexicano (Stahle et al., 2016). Los autores reconstruyeron la precipitación para el periodo enero-junio partir de información meteorológica e información de anillos de crecimiento de Pseuodotsuga menziesii en Chihuahua, México (Castruita-Esparza et al., 2016). Las líneas negra, azul y roja representan precipitación reconstruida, precipitación media anual y curva suavizada, respectivamente. El punto negro muestra la localización del área de estudio.

puede representar hasta cuatro años de retroceso en la captura de carbono (Ciais, et al., 2005). Estudios en bosques de coníferas del norte de México muestran que los retardos en crecimiento radial (histéresis) son de 4 a 10 años por efecto de sequía, lo cual afecta temporalmente la capacidad de captura de carbono y las estimaciones confiables del aprovechamiento forestal (Castruita et al., 2019).

\section{CONCLUSIONES}

Es importante comprender las leyes que definen el comportamiento de los ecosistemas forestales para anticipar la toma de decisiones ante el cambio climático. La mayor pérdida neta de superficie forestal y de carbono del ecosistema se origina en las regiones tropicales; $\sin$ embargo, los bosques secundarios del trópico, por su alta tasa de crecimiento, son parte de la solución para mitigar las emisiones de $\mathrm{CO}_{2}$. La pérdida de superficie forestal provocada por el cambio climático significa la pérdida de los servicios ambientales, como el agua y la biodiversidad. La regulación estomática de las especies forestales determina el balance de agua y carbono en la vegetación, y por lo tanto, el intercambio de gases entre la hoja y la atmósfera. La consideración más detallada del funcionamiento de los ecosistemas forestales permitirá generar modelos con mejor capacidad de predicción de captura de carbono ante el escenario de cambio climático. En términos de la formación de la madera, sus componentes deben satisfacer eficiencia en soporte y transporte de agua; sin embargo, la formación de dichos componentes es en gran medida una función de las condiciones del clima y su variación. El cambio climático está afectando la fisiología de los árboles y los componentes de resistencia, resiliencia e histéresis de los bosques.

\section{AGRADECIMIENTO}

Al Dr. Abel Muñoz Orozco ('Descanse en paz) por motivar al primer autor a escribir el presente trabajo. A los revisores anónimos, su trabajo fue muy importante para llegar a una versión final. 


\section{BIBLIOGRAFÍA}

Allen C. D., D. D. Breshears and N. G. McDowell (2015) On underestimation of global vulnerability to tree mortality and forest die-off from hotter drought in the anthropocene. Ecosphere 6:129, https:// doi.org/10.1890/ES15-00203.1

Anderegg W. R., C. Schwalm, F. Biondi, J. J. Camarero, G. Koch, M. Litvak, ... and A. Williams (2015) Pervasive drought legacies in forest ecosystems and their implications for carbon cycle models. Science 349:528-532, https://doi.org/10.1126/science. aab1833

Anderegg W. R., T. Klein, M. Bartlett, L. Sack, A. F. Pellegrini, B. Choat and S. Jansen (2016) Meta-analysis reveals that hydraulic traits explain cross-species patterns of drought-induced tree mortality across the globe. Proceedings of the National Academy of Sciences of the United States of America 113:5024-5029, https://doi.org/10.1073/pnas.1525678113

Battipaglia G., M. Saurer, P. Cherubini, C. Calfapietra, H. R. McCarthy, R. J. Norby and M. F. Cotrufo (2013) Elevated $\mathrm{CO}_{2}$ increases treelevel intrinsic water use efficiency: insights from carbon and oxygen isotope analyses in tree rings across three forest face sites. New Phytologist 197:544-554, https://doi.org/10.1111/ nph. 12044

Begum S., K. Kudo, M. H. Rahman, S. Nakaba, Y. Yamagishi, E. Nabeshima, ... and R. Funada (2018) Climate change and the regulation of wood formation in trees by temperature. Trees 32:3-15

Bonan G. B. (2008) Forests and climate change: forcings, feedbacks, and the climate benefits of forests. Science 320:1444-1449, https://doi.org/10.1126/science.1155121

Castruita-Esparza L. U., A. Correa-Díaz, A. Gómez-Guerrero, J. VillanuevaDíaz, M. E. Ramírez-Guzmán, A. Velázquez-Martínez and G. ÁngelesPérez (2016) Basal area increment series of dominant trees of Pseudotsuga menziesii (Mirb.) Franco show periodicity according to global climate patterns. Revista Chapingo Serie Ciencias Forestales y del Ambiente 22:380-397, https://doi. org/10.5154/r.rchscfa.2015.10.048

Castruita-Esparza L. U., L. C. R. Silva, A. Gómez-Guerrero, J. Villanueva-Díaz, A. Correa-Díaz and W. R. Horwath (2019) Coping with extreme events: growth and water-use efficiency of trees in western Mexico during the driest and wettest periods of the past one hundred sixty years. JGR Biogeosciences 124:3419-3431, https://doi.org/10.1029/2019JG005294

Chen F., M. Opała-Owczarek, P. Owczarek and Y. Chen (2020) Summer monsoon season streamflow variations in the middle Yellow river since 1570 CE inferred from tree rings of Pinus tabulaeformis. Atmosphere 11:717, https://doi.org/10.3390/ atmos 11070717

Choat B., T. J. Brodribb, C. R. Brodersen, R. A. Duursma, R. Lopez and B. E. Medlyn (2018) Triggers of tree mortality under drought. Nature 558:531-539, https://doi.org/10.1038/s41586-018-0240-x

Chazdon R. L., E. N. Broadbent, D. M. Rozendaal, F. Bongers, A. M. A. Zambrano, T. M. Aide, ... and L. Poorter (2016) Carbon sequestration potential of second-growth forest regeneration in the Latin American tropics. Science Advances 2:e1501639, https://doi. org/10.1126/sciadv.1501639

Ciais P., M. Reichstein, N. Viovy, A. Granier, J. Ogée, V. Allard, ... and R. Valentini (2005) Europe-wide reduction in primary productivity caused by the heat and drought in 2003. Nature 437:529-533, https://doi. org/10.1038/nature03972

CONAFOR (2019) El Sector Forestal Mexicano en Cifras 2019. Bosques para el Bienestar Social y Climático. Comisión Nacional Forestal. Zapopa, Jalisco, México. 104 p.

CorreaDíaz A., L. Silva, W. Horwath, A. GómezGuerrero, J. Vargas Hernández, J. VillanuevaDíaz, ... and J. SuárezEspinoza (2019) Linking remote sensing and dendrochronology to quantify climate induced shifts in high elevation forests over space and time. JGR Biogeosciences 124:166-183, https://doi. org/10.1029/2018JG004687

Correa-Díaz A., L. C. R. Silva, W. R. Horwath, A. Gómez-Guerrero, J. Vargas-Hernández, J. Villanueva-Díaz, ... and A. VelázquezMartínez (2020a) From trees to ecosystems: spatiotemporal scaling of climatic impacts on montane landscapes using dendrochronological, isotopic, and remotely sensed data.
Global Biogeochemical Cycles 34:e2019GB006325, https://doi org/10.1029/2019GB006325

Correa-Díaz A., A. Gómez-Guerrero, J. J. Vargas-Hernández, P. Rozenberg and W. R. Horwath (2020b) Long-term wood micro-density variation in alpine forests at central México and their spatial links with remotely sensed information. Forests 11:452, https://doi. org/10.3390/f11040452

Curtis P. G., C. M. Slay, N. L. Harris, A. Tyukavina and M. C. Hansen (2018) Classifying drivers of global forest loss. Science 361:11081111, https://doi.org/10.1126/science.aau3445

Dawson T. E., S. Mambelli, A. H. Plamboeck, P. H. Templer and K. P. Tu (2002) Stable isotopes in plant ecology. Annual Review of Ecology and Systematics 33:507-559, https://doi.org/10.1146/annurev ecolsys.33.020602.095451

Duffy K. A., C. R. Schwalm, V. L. Arcus, G. W. Koch, L. L. Liang and L. A. Schipper (2021) How close are we to the temperature tipping point of the terrestrial biosphere? Science Advances 7:eaay1052, https:// doi.org/10.1126/sciadv.aay 1052

FAO, Food and Agriculture Organization (2020) Global Forest Resources Assessment 2020: Main Report. Rome, Italy. 184 p.

Farquhar G. D., M. O'leary and J. Berry (1982) On the relationship between carbon isotope discrimination and the intercellular carbon dioxide concentration in leaves. Functional Plant Biology 9:121 137, https://doi.org/10.1071/PP9820121

Fei S., J. M. Desprez, K. M. Potter, I. Jo, J. A. Knott and C. M. Oswalt (2017) Divergence of species responses to climate change. Science Advances 3:e1603055, https://doi.org/10.1126/sciadv.1603055

Gómez-Guerrero A. and T. Doane (2018) The response of forest ecosystems to climate change. In: Climate Change Impacts on Soil Processes and Ecosystem Properties. W. R. Horwath and K. Yakov (eds.). Elsevier. Amsterdam, The Netherlands. pp:185206, https://doi.org/10.1016/B978-0-444-63865-6.00007-7

Gómez-Guerrero A., L. C. R. Silva, M. Barrera-Reyes, B. Kishchuk, A. VelazquezMartinez, T. Martinez-Trinidad, ... and W. R. Horwath (2013) Growth decline and divergent tree ring isotopic composition $\left({ }^{13} \mathrm{C}\right.$ and ${ }^{18} \mathrm{O}$ ) contradict predictions of $\mathrm{CO}_{2}$ stimulation in high altitudinal forests. Global Change Biology 19:1748-1758, https://doi org/10.1111/gcb.12170

Hansen M. C., P. V. Potapov, R. Moore, M. Hancher, S. Turubanova, A. Tyukavina, ... and T. Loveland (2013) High-resolution global maps of 21stcentury forest cover change. Science 342:850-853, https://doi org/10.1126/science.1244693

Huang K., C. X. Yi, D. Wu, T. Zhou, X. Zhao, W. J. Blanford, ... and Z. Li (2015) Tipping point of a conifer forest ecosystem under severe drought. Environmental Research Letters 10:031002, https:// doi.org/10.1088/1748-9326/10/2/024011

IPCC, Grupo Intergubernamental de Expertos sobre el Cambio Climático (2018) Calentamiento Global de $1,5{ }^{\circ} \mathrm{C}$, Informe Especial del IPCC sobre los Impactos del Calentamiento Global de $1,5^{\circ} \mathrm{C}$ con Respecto a los Niveles Preindustriales y las Trayectorias Correspondientes que Deberían Seguir las Emisiones Mundiales de Gases de Efecto Invernadero, en el Contexto del Reforzamiento de la Respuesta Mundial a la Amenaza del Cambio Climático, el Desarrollo Sostenible y los Esfuerzos por Erradicar la Pobreza. Resumen para Responsables de Políticas. V. Masson-Delmotte, P. Zhai, P. Hans-Otto, D. Roberts, J. Skea P. R. Shukla, ... y T. Waterfield (eds.). Grupo Intergubernamental de Expertos sobre el Cambio Climático. Ginebra, Suiza. 26 p.

Jump A. S., C. Mátyás and J. Peñuelas (2009) The altitude-for-latitude disparity in the range retractions of woody species. Trends in Ecology \& Evolution 24:694-701, https://doi.org/10.1016/j. tree.2009.06.007

Kannenberg S. A., J. T. Maxwell, N. Pederson, L. D'Orangeville, D. L. Ficklin and R. P. Phillips (2019) Drought legacies are dependent on water table depth, wood anatomy and drought timing across the eastern US. Ecology Letters 22:119-127, https://doi org/10.1111/ele.13173

Keenan R. J., G. A. Reams, F. Achard, J. V. de Freitas, A. Grainger and E. Lindquist (2015) Dynamics of global forest area: results from the FAO Global Forest Resources Assessment 2015. Forest Ecology and Management 352:9-20, https://doi.org/10.1016/j. foreco.2015.06.014

Lloret F., E. G. Keeling and A. Sala (2011) Components of tree resilience: 
effects of successive low-growth episodes in old ponderosa pine forests. Oikos 120:1909-1920, https://doi.org/10.1111/ j.1600-0706.2011.19372.x

Masum K. M., A. Mansor, S. A. M. Sah and H. San Lim (2017) Effect of differential forest management on land-use change (LUC) in a tropical hill forest of Malaysia. Journal of Environmental Management 200:468-474, https://doi.org/10.1016/j. jenvman.2017.06.009

Mantero-García H. D., A. Gómez-Guerrero, F. Gavi-Reyes, B. P. Zamora-Morales and C. Ramírez-Ayala (2019) ¿Es sustentable el aprovechamiento de tierra de hoja en bosques de encino?. Madera y Bosques 25: e2531807, https://doi.org/10.21829/myb.2019.2531807

McCarroll D. and N. J. Loader (2004) Stable isotopes in tree rings. Quaternary Science Reviews 23:771-801, https://doi. org/10.1016/j.quascirev.2003.06.017

McDowell N. G. and C. D. Allen (2015) Darcy's law predicts widespread forest mortality under climate warming. Nature Climate Change 5:669-672, https://doi.org/10.1038/nclimate2641

McDowell N. G., S. White and W. T. Pockman (2008) Transpiration and stomatal conductance across a steep climate gradient in the southern rocky mountains. Ecohydrology 1:193-204, https:// doi.org/10.1002/eco.20

Mendivelso H. A., J. J. Camarero and E. Gutiérrez (2016) Dendrocronología en bosques neotropicales secos: métodos, avances y aplicaciones. Ecosistemas 25:66-75, https://doi.org/10.7818/ ECOS.2016.25-2.08

Otto J., D. Berveiller, F.-M. Bréon, N. Delpierre, G. Geppert, A. Granier, ... and B. Longdoz (2014) Forest summer albedo is sensitive to species and thinning: how should we account for this in earth system models? Biogeosciences 11:2411-2427, https://doi. org/10.5194/bg-11-2411-2014

Pan Y., R. A. Birdsey, J. Fang, R. Houghton, P. E. Kauppi, W. A. Kurz, ... and J. G. Canadell (2011) A large and persistent carbon sink in the world's forests. Science 333:988-993, https://doi.org/10.1126/ science.1201609

Peñuelas J., J. G. Canadell and R. Ogaya (2011) Increased water-use efficiency during the 20th century did not translate into enhanced tree growth. Global Ecology and Biogeography 20:597-608, https://doi.org/10.1111/j.1466-8238.2010.00608.x

Peñuelas J., P. Ciais, J. G. Canadell, I. A. Janssens, M. Fernández-Martínez, J. Carnicer, ... and J. Sardans (2017) Shifting from a fertilizationdominated to a warming-dominated period. Nature Ecology \& Evolution 1:1438-1445, https://doi.org/10.1038/s41559-0170274-8

Piao S., X. Wang, T. Park, C. Chen, X. Lian, Y. He, ... and R. B. Myneni (2020) Characteristics, drivers and feedbacks of global greening. Nature Reviews Earth and Environment 1:14-27, https://doi. org/10.1038/s43017-019-0001-x

Plomion C., G. Leprovost and A. Stokes (2001) Wood formation in trees. Plant Physiology 127:1513-1523, https://doi.org/10.1104/ pp.010816

Roden J. and G. D. Farquhar (2012) A controlled test of the dual-isotope approach for the interpretation of stable carbon and oxygen isotope ratio variation in tree rings. Tree Physiology 32:490-503, https://doi.org/10.1093/treephys/tps019

Saurer M., R. T. Siegwolf and F. H. Schweingruber (2004) Carbon isotope discrimination indicates improving water-use efficiency of trees in northern Eurasia over the last 100 years. Global Change Biology 10:2109-2120, https://doi.org/10.1111/j.13652486.2004.00869.x
Schlesinger W. H. and E. S. Bernhardt (2013) Biogeochemistry: An Analysis of Global Change. Academic Press. San Diego, California, USA. $672 \mathrm{p}$

Seidl R., D. Thom, M. Kautz, D. Martin-Benito, M. Peltoniemi, G. Vacchiano, ... and C. P. 0. Reyer (2017) Forest disturbances under climate change. Nature Climate Change 7:395-402, https://doi. org/10.1038/nclimate3303

Stahle D. W., E. R. Cook, D. J. Burnette, J. Villanueva, J. Cerano, J. N. Burns, and M. C. Torbenson (2016) The Mexican drought atlas: treering reconstructions of the soil moisture balance during the late pre-hispanic, colonial, and modern eras. Quaternary Science Reviews 149:34-60, https://doi.org/10.1016/j. quascirev.2016.06.018

Stovall A. E. L., H. Shugart and X. Yang (2019) Tree height explains mortality risk during an intense drought. Nature Communications 10:4385, https://doi.org/10.1038/s41467-019-12380-6

Varkkey H., A. Tyson and S. A. B. Choiruzzad (2018) Palm oil intensification and expansion in Indonesia and Malaysia: environmental and socio-political factors influencing policy. Forest Policy and Economics 92:148-159, https://doi.org/10.1016/j. forpol.2018.05.002

Villanueva-Díaz J., B. H. Luckman, D. W. Stahle, M. D. Therrell, M. K. Cleaveland, J. Cerano-Paredes and R. Jasso-Ibarra (2005) Hydroclimatic variability of the upper Nazas basin: water management implications for the irrigated area of the Comarca Lagunera, Mexico. Dendrochronologia 22:215-223, https://doi. org/10.1016/j.dendro.2005.04.005

Villanueva J., A. Gómez, J. Cerano, S. Rosales, J. Estrada, L. U. Castruita and A. R. Martínez (2017) La variabilidad del caudal del río Acaponeta inferida mediante series de anillos de crecimiento en coníferas. Tecnología y Ciencias del Agua 8:55-74, https:// doi.org/10.24850/j-tyca-2017-03-04

Voltas J., J. J. Camarero, D. Carulla, M. Aguilera, A. Ortiz and J. P. Ferrio (2013) A retrospective, dual-isotope approach reveals individual predispositions to winter-drought induced tree dieback in the southernmost distribution limit of Scots pine. Plant, Cell \& Environment 36:1435-1448, https://doi.org/10.1111/ pce. 12072

Voelker S. L., J. R. Brooks, F. C. Meinzer, R. Anderson, M. K. F. Bader, G. Battipaglia, ... and L. Wingate (2016) A dynamic leaf gas exchange strategy is conserved in woody plants under changing ambient $\mathrm{CO}_{2}$ : evidence from carbon isotope discrimination in paleo and $\mathrm{CO}_{2}$ enrichment studies. Global Change Biology 22:889-902, https://doi.org/10.1111/gcb.13102

Xu Y., W. Li, X. Shao, Z. Xu and P. Nugroho (2014) Long-term trends in intrinsic water-use efficiency and growth of subtropical Pinus tabulaeformis Carr. and Pinus taiwanensis Hayatain in central China. Journal of Soils and Sediments 14:917-927, https://doi. org/10.1007/s11368-014-0878-4

Yang S., L. Lei, Z. Zeng, Z. He and H. Zhong (2019) An assessment of anthropogenic $\mathrm{CO}_{2}$ emissions by satellite-based observations in China. Sensors 19:1118, https://doi.org/10.3390/s19051118

Zhang M., N. Liu, R. Harper, Q. Li, K. Liu, X. Wei, ... and S. Liu (2017) A global review on hydrological responses to forest change across multiple spatial scales: importance of scale, climate, forest type and hydrological regime. Journal of Hydrology 546:44-59, https://doi.org/10.1016/j.jhydrol.2016.12.040

Zhu Z., S. Piao, R. B. Myneni, M. Huang, Z. Zeng, J. G. Canadell, ... and N. Zeng (2016) Greening of the earth and its drivers. Nature Climate Change 6:791-795, https://doi.org/10.1038/nclimate3004 